ARTIGO

Recebido em: 01/09/2016

Aceito em: 10/04/2017

\title{
Colaboração da Matemática brasileira na Web of Science (2004-2013)
}

Collaboration of Brazilian Mathematics in Web of Science (2004-2013)

\author{
Daniela Gralha de Caneda QUEIROZ (daniela.caneda@gmail.com)* \\ Ana Maria Mielniczuk de MOURA (ana.moura@ufrgs.br)** \\ * Doutoranda em Ciência da Informação PPGCINF/FCI - UnB. \\ ** Professora da Faculdade de Biblioteconomia e Comunicação - UFRGS.
}

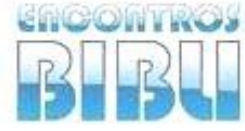

v. 22, n. $49,2017$.

p. $115-126$

ISSN 1518-2924

\section{Resumo}

Pesquisa bibliométrica cujo objetivo é analisar as características da colaboração da matemática brasileira, através dos artigos indexados na base de dados Web of Science, no período de 2004 a 2013. Os dados foram analisados sob a figura de tabelas, gráficos e grafos gerados através do uso dos softwares Excel, BibExcel, UCINET e NETDRAW. Apresenta como resultados a recuperação de 8.625 artigos. Constata a presença de 75 países e 1.342 instituições. Identifica que há $14,04 \%$ de autoria única, 34,91\% de colaborações nacionais e $51,04 \%$ de colaborações internacionais. Conclui que a área encontra-se em franco desenvolvimento, com significativa internacionalização.

Palavras-chave: Colaboração Científica; Matemática; Brasil

\begin{abstract}
Bibliometric research which aims to analyze the characteristics of scientific collaboration on Brazilian mathematics, through the articles indexed in the database of Web of Science, from 2004 to 2013. The data were analyzed under the figure of tables, charts and graphs generated through the use of Excel, BibExcel, UCINET and NETDRAW. It presents results as the recovery of 8.625 articles. It is noticed the presence of 75 countries and 1342 . It identifies that there are a $14.04 \%$ percentage of single authorship, $34.91 \%$ of national collaborations and $51.04 \%$ of international collaborations. It concludes that the area is rapidly developing, with significant internationalization.
\end{abstract}

Keywords: Scientific Collaboration; Math; Brazil

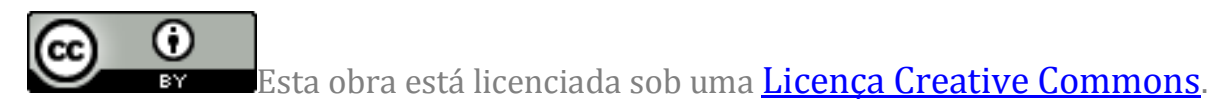




\section{INTRODUÇÃo}

No ano de 2010 o pesquisador brasileiro Jacob Palis, do Instituto Nacional de Matemática Pura e Aplicada - IMPA, foi vencedor do prêmio Balzan, concedido anualmente a pesquisadores internacionais das áreas de ciências humanas e naturais, artes e cultura, pela Fondazione Internazionale Premio Balzan, fundação ítalo-suíça. No ano de 2014 o matemático brasileiro Artur Avila Cordeiro de Melo, também do IMPA, recebeu a Medalha Fields, considerada como se fosse o Nobel de matemática, tornando-se o primeiro cientista latino-americano e lusófono a conquistar tal medalha.

Documento de Área 2013 da Coordenação de Aperfeiçoamento de Pessoal de Nível Superior - CAPES (2014) traz que a área de matemática tem ocupado lugar de destaque no cenário internacional, pois, além de participar do International Mathematical Union - IMU, tem apresentado muitos pesquisadores como conferencistas em eventos internacionais e como membros de corpos editoriais de periódicos. Soma-se a isso os atos de cooperação internacional com a National Science Foundation - NSF (Estados Unidos), German National Research Center for Information Technology (Alemanha) e Ministère des Affaires étrangères (França), com pesquisas laboratoriais e de simulação computacional em áreas como a do petróleo e a do clima (SIMIS et al., 2002).

Esse panorama mostra que a matemática brasileira se encontra em franco desenvolvimento, rumo à excelência em nível mundial. Diante disso, faz-se a importância de um estudo na área, a fim de mapear esse campo de pesquisa, descobrindo sua estrutura e suas relações, como suporte às políticas científicas. Sanz-Casado (2014) observa que ao se mapear um campo, pode-se, por exemplo, modernizar uma instituição, reduzir gastos e pesquisar áreas mais interessantes. Desse modo, esse estudo tem como objetivo analisar a colaboração da matemática brasileira através dos artigos de periódicos indexados na Web of Science - WoS, entre os anos de 2004 a 2013.

\section{PROCEDIMENTOS METODOLÓGICOS}

Essa pesquisa é do tipo bibliométrica, descritiva, com abordagem quantitativa, de natureza aplicada e em nível macro, tendo em vista que se analisou a colaboração científica brasileira em matemática, através dos artigos indexados na base de dados WoS, no período de 2004 a 2013.

A pesquisa bibliométrica, segundo Leydesdorff (c2001), tem sido utilizada nas políticas nacionais de ciência, permitindo verificar os pontos fortes e fracos da pesquisa. Macias-Chapula (1998) afirma que ela serve também para situar uma nação em relação ao restante do mundo, uma instituição em relação a sua nação e um pesquisador em relação a sua comunidade científica; além disso, auxilia na avaliação da ciência, na tomada de decisões e no gerenciamento da pesquisa.

A base de dados WoS, da Thomson Reuters Scientific, foi escolhida em função da sua cobertura, credibilidade e usabilidade. Glänzel (2003) afirma que a WoS tornou-se fonte básica para análises bibliométricas, sendo considerada como requisito fundamental neste tipo de estudo.

A estratégia de busca para essa pesquisa foi: utilização da "pesquisa avançada" da WoS; uso do rótulo de campo "CU=(Brasil OR Brazil)", para recuperação de todos os registros com vínculo com alguma instituição brasileira; atribuição do operador booleano "AND" entre os rótulos de campo "CU" e "WC"; aplicação das categorias de assunto no campo "WC=(operations research \& management science $\mathrm{OR}$ mathematics, applied OR mathematics, interdisciplinary applications OR mathematics)"; emprego da opção "All languages" em idiomas, pretendendo recuperar todos os documentos; restrição do tipo de documento a "Article", considerado como principal canal de comunicação científica da ciência e principal meio para se estudar bibliometria; utilização dos anos "de 2004 até 2013" em tempo estipulado; atribuição das coleções 
Science Citation Index Expanded (SCI-EXPANDED) e Social Sciences Citation Index (SSCI) à pesquisa.

Nas análises dos dados foram utilizados os softwares BibExcel, Excel, UCINET e NETDRAW. Para a complementação dessas análises foram ainda consultados: Journal Citations Reports (JCR) e Plataforma Lattes, do Conselho Nacional de Desenvolvimento Científico e Tecnológico - CNPq.

\section{APRESENTAÇÃO E DISCUSSÃO DOS RESULTADOS}

Os critérios predeterminados para a coleta de dados permitiram a recuperação de 8.625 artigos da área de matemática em que pelo menos um dos autores fosse vinculado a alguma instituição brasileira entre os anos de 2004 e 2013. E a fim de se investigar a colaboração científica na área da matemática brasileira, realizou-se a análise de coautoria. Assim, uma primeira análise permitiu que se chegasse ao gráfico abaixo:

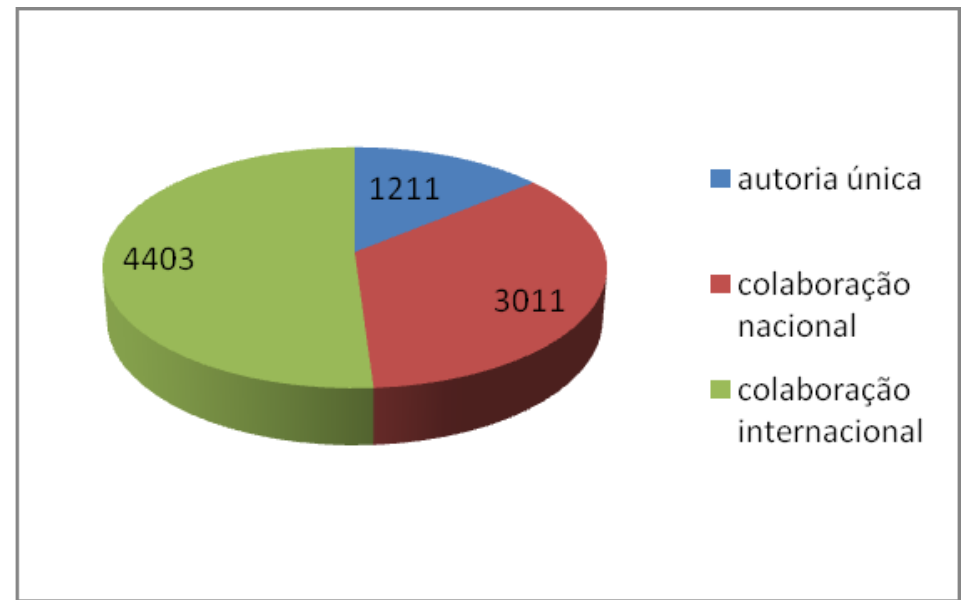

Gráfico 1: Número de artigos conforme o tipo de colaboração dos artigos brasileiros da área de matemática indexados na WoS entre 2004 e $2013(n=8.625)$

Fonte: Dados de pesquisa.

O Gráfico 1 mostra o número de autorias únicas (14,04\%) e também traz os números de colaborações tanto nacionais quanto internacionais. As colaborações internacionais são em maior número, com 51,04\%. Porcentagem essa maior que a identificada por Castanha e Grácio (2013), que encontraram 38,9\% de colaboração internacional na área de matemática entre 2002 e 2011 na base Scopus, e por Vanz (2009), que encontrou cerca de 40\%, entre 2004 e 2006, na WoS. Já as colaborações nacionais possuem $34,91 \%$ do total.

As colaborações nacionais e internacionais deram um total relevante de 85,95\% (59,39\% de colaborações internacionais e 40,61\% de colaborações nacionais). Esses números indicam que há uma significativa internacionalização da área e que a colaboração é predominante na sua produção científica. Tal resultado vai ao encontro do resultado encontrado por Vanz (2009), em que a matemática é caracterizada pela baixa colaboração nacional e alta colaboração internacional, devido ao pequeno tamanho da área, a qual incentiva seus pesquisadores a buscar parceiros na comunidade internacional. No entanto, Vanz $(2009$, p.128) encontrou "percentuais de coautoria inexpressivos" em sua pesquisa, diferentemente do encontrado aqui.

Abaixo é exibido o Gráfico 2 o qual apresenta os níveis de colaboração entre os países, totalizado por 4.403 artigos: 


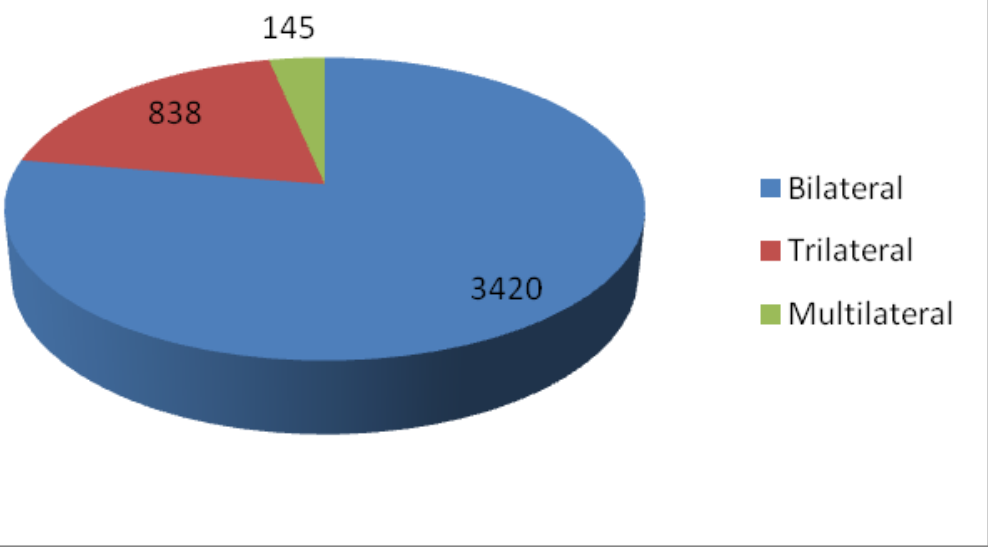

Gráfico 2: Níveis de colaboração conforme o número de países colaboradores dos artigos brasileiros da área de matemática indexados na WoS entre 2004 e $2013(n=4.403)$

Fonte: Dados de pesquisa.

Em tratando-se da quantidade de países por artigo analisado, o nível de colaboração bilateral é o que mais predomina, ocupando 77,67\%. Em seguida vem o nível de colaboração trilateral, com 19,03\%. Já o nível de colaboração multilateral (mais de três países) ocupa apenas 3,29\%, confirmando a característica da área de matemática de não trabalhar em grupos grandes.

Logo mais é apresentada a Tabela 1 com o detalhamento do Gráfico 2, com o número de países que colaboraram com o Brasil (75 países colaboradores no total). E um exame na Tabela 1 corrobora que 51,04\% dos 8.625 artigos foram escritos em parceria entre o Brasil e outra nação:

Tabela 1: Número de países por artigos brasileiros da área de matemática indexados na WoS entre 2004 e $2013(n=4.403)$

\begin{tabular}{lcc}
\hline \multicolumn{1}{c}{ № países } & № artigos & \% \\
\hline Brasil mais 1 país & 3.420 & $77,67 \%$ \\
\hline Brasil mais 2 países & 838 & $19,03 \%$ \\
\hline Brasil mais 3 países & 129 & $2,93 \%$ \\
\hline Brasil mais 4 países & 14 & $0,32 \%$ \\
\hline Brasil mais 5 países & 1 & $0,02 \%$ \\
\hline Brasil mais 9 países & 1 & $0,02 \%$ \\
\hline \multicolumn{1}{c}{ Total } & $\mathbf{4 . 4 0 3}$ & $\mathbf{1 0 0 , 0 0 \%}$ \\
\hline
\end{tabular}

Fonte: Dados de pesquisa.

A seguir é mostrada a Tabela 2, em que são apresentados os principais países, com seus números de instituições (1.342 no total) e de artigos. 
Tabela 2: Número de instituições e de artigos por país dos autores dos artigos brasileiros da área de matemática indexados na WoS entre 2004 e $2013(\mathrm{n}=1.342)$

\begin{tabular}{lccccc}
\hline \multicolumn{1}{c}{ País } & $\mathbf{N o}$ instituições & $\mathbf{\% ( 1 . 3 4 2 )}$ & $\mathbf{N o}$ artigos & $\mathbf{\% ~ ( 8 . 6 2 5 )}$ & Média art./inst. \\
\hline Brasil & 306 & 22,80 & 8.625 & 100,00 & 28,19 \\
\hline EUA & 194 & 14,46 & 803 & 9,31 & 4,14 \\
\hline França & 107 & 7,97 & 580 & 6,72 & 5,42 \\
\hline Alemanha & 60 & 4,47 & 212 & 2,46 & 3,53 \\
\hline Itália & 53 & 3,95 & 225 & 2,61 & 4,25 \\
\hline China & 53 & 3,95 & 89 & 1,03 & 1,68 \\
\hline Espanha & 51 & 3,80 & 435 & 5,04 & 8,53 \\
\hline Inglaterra & 40 & 2,98 & 194 & 2,25 & 4,85 \\
\hline Canadá & 36 & 2,68 & 192 & 2,23 & 5,33 \\
\hline Japão & 26 & 1,94 & 58 & 0,67 & 2,23 \\
\hline Índia & 26 & 1,94 & 40 & 0,46 & 1,54 \\
\hline Portugal & 24 & 1,79 & 188 & 2,18 & 7,83 \\
\hline Chile & 22 & 1,64 & 245 & 2,84 & 11,14 \\
\hline Rússia & 19 & 1,42 & 123 & 1,43 & 6,47 \\
\hline Polônia & 19 & 1,42 & 59 & 0,68 & 3,11 \\
\hline Argentina & 18 & 1,34 & 119 & 1,38 & 6,61 \\
\hline Colômbia & 18 & 1,34 & 55 & 0,64 & 3,06 \\
\hline México & 15 & 1,12 & 69 & 0,80 & 4,60 \\
\hline Austrália & 14 & 1,04 & 50 & 0,58 & 3,57 \\
\hline Holanda & 0,82 & 33 & 0,38 & 3,00 \\
\hline Fonte: Dad & & & & \\
\hline
\end{tabular}

Fonte: Dados de pesquisa.

O país que mais colaborou com o Brasil foram os Estados Unidos, com uma fatia de 9,31\% do total, seguido da França, com 6,72\%, e da Espanha, com 5,04\%. Pesquisa de Moura et al. (2015, p.80) sobre a produção conjunta entre o Brasil e a Espanha na WoS, entre os anos de 2006 e 2012, constatou que a área da matemática "é um ponto forte da colaboração bilateral, já que conta com um elevado índice de atividade. [. . .] ambos são potentes nela e se complementam através da colaboração." Após, essas colaborações caem para menos de 3\%. Percebe-se que a proximidade física não é um fator determinante para a colaboração entre o Brasil e esses países, pois o Chile é o país próximo com maior colaboração e, no entanto, está presente em somente $2,84 \%$ da produção de artigos. Os outros países mais próximos são Argentina com 1,38\% de colaboração; Colômbia, com 0,64\%; Uruguai, com 0,43\%; Equador, com 0,02\%; e Paraguai, com 0,02\%. Contudo, todos apresentam porcentagem baixa de colaboração.

Em seguida é apresentada a Figura 1, com a rede formada a partir da colaboração entre esses países: 


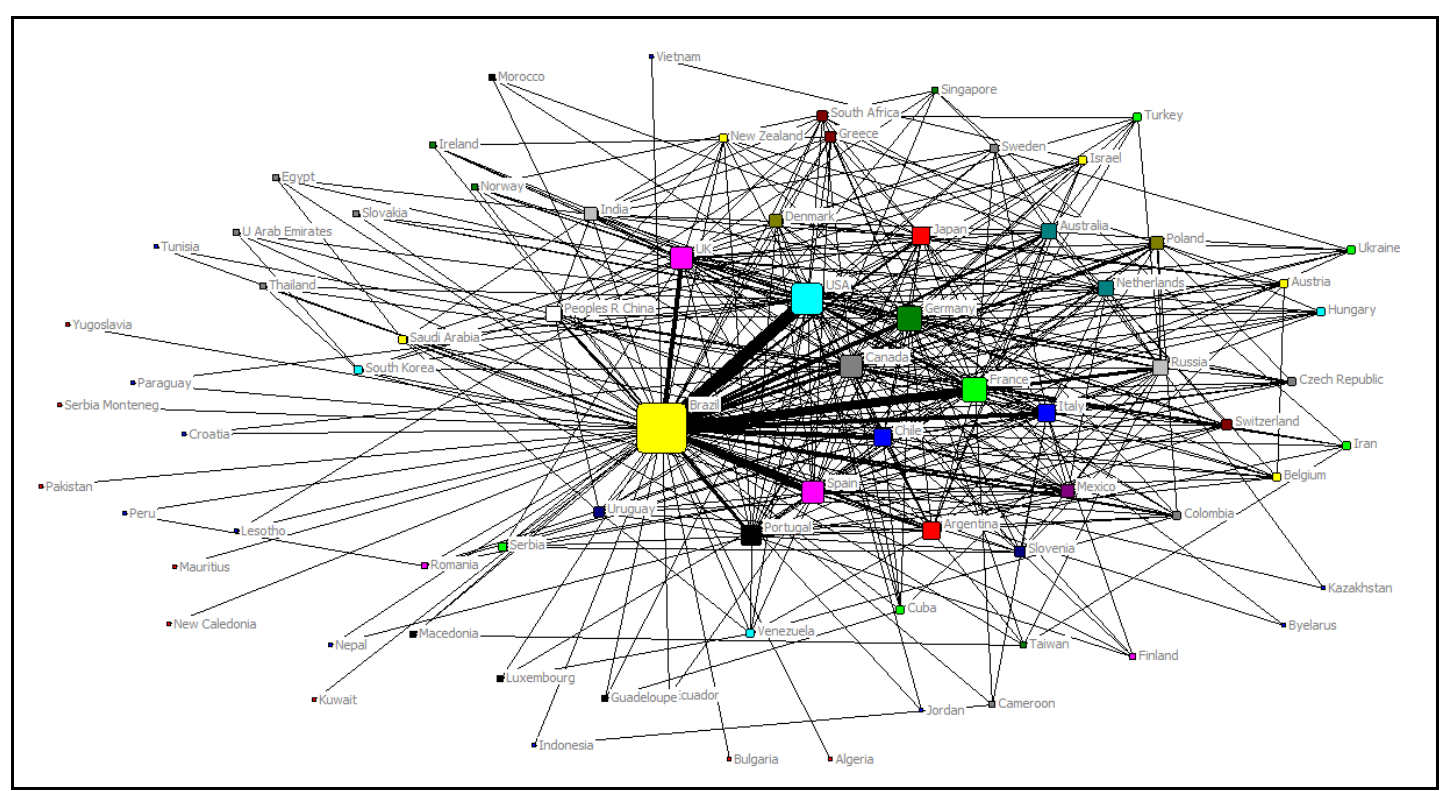

Figura 1 - Mapa de colaboração dos países presentes nos artigos brasileiros da área de matemática indexados na WoS entre 2004 e 2013

Fonte: Dados de pesquisa.

Nota: Por especificações do software utilizado, os nomes dos países estão em inglês.

Esse mapa de colaboração é formado por 76 nós ou países, os quais apresentaram desde o mínimo de uma ligação até o máximo de 75 ligações, totalizando 830 ligações. Os maiores clusters, os quais foram aqui analisados, são Brasil, Estados Unidos e França.

0 maior cluster é representado pelo Brasil (nó na cor amarela), com 75 ligações. Suas ligações mais fortes (linhas mais grossas no mapa) são com os Estados Unidos (nó na cor azul clara), França (nó na cor verde limão) e Espanha (nó na cor rosa).

O segundo maior cluster representa os Estados Unidos, com 46 ligações. Além do Brasil, os Estados Unidos relacionam-se principalmente com França (45 artigos em comum) e Reino Unido (29 artigos em comum). 0 terceiro maior cluster representa a França, com 35 ligações. Além do Brasil e dos Estados Unidos, a França relaciona-se também com o Canadá (nó na cor cinza chumbo).

Castanha e Grácio (2013) colocam que a área da matemática é marcada por forte colaboração internacional, em virtude da sua linguagem ser universal e dos recursos materiais para pesquisa serem menos dispendiosos. 0 estudo das autoras teve como objetivo analisar a contribuição científica brasileira na área da matemática, através dos artigos publicados em periódicos indexados pela Scopus, no período de 2002 a 2011. Nesse período analisado, na área da matemática, o Brasil colaborou com outras 85 nações. Os seis primeiros países com os quais o Brasil mais colaborou estão entre os 10 mais produtivos pelo ranking da Scopus. Só os Estados Unidos possuíam $10 \%$ de colaboração com o Brasil; os outros países são: França $(6,6 \%)$, Alemanha (3,9\%), Reino Unido (3,7\%), Espanha (3,6\%), Itália $(3,3 \%)$, Chile $(2,4 \%)$, Rússia $(2,4 \%)$, Canadá $(2,3 \%)$, Portugal $(2,1 \%)$ e Argentina $(1,8 \%)$. Assim como na pesquisa de Castanha e Grácio, aqui os Estados Unidos e a França foram os países que mais colaboraram com o Brasil. Em outro estudo realizado por Castanha, Hilário e Grácio (2013), as colaborações científicas internacionais corresponderam a $40 \%$ dos artigos publicados pelo Brasil, em pesquisa da produção científica em matemática com presença de pesquisadores brasileiros, espanhóis e portugueses, na base Scopus, no período de 2001 a 2010.

Abaixo tem-se a representação da Tabela 3, em que consta o número de diferentes instituições por número de artigos publicados: 
Tabela 3: Número de instituições distribuídas por ano dos artigos brasileiros da área de matemática indexados na WoS entre 2004 e 2013

\begin{tabular}{|c|c|c|c|c|c|c|c|c|c|c|c|c|}
\hline $\begin{array}{c}\text { № } \\
\text { Inst. }\end{array}$ & $\begin{array}{c}200 \\
4 \\
\end{array}$ & $\begin{array}{c}200 \\
5\end{array}$ & $\begin{array}{c}200 \\
6\end{array}$ & $\begin{array}{c}200 \\
7 \\
\end{array}$ & $\begin{array}{c}200 \\
8 \\
\end{array}$ & $\begin{array}{c}200 \\
9 \\
\end{array}$ & $\begin{array}{c}201 \\
0\end{array}$ & $\begin{array}{c}201 \\
1 \\
\end{array}$ & $\begin{array}{c}201 \\
2 \\
\end{array}$ & $\begin{array}{c}201 \\
3 \\
\end{array}$ & $\begin{array}{l}\text { Tot. } \\
\text { Art. }\end{array}$ & $\%$ \\
\hline 1 & 339 & 364 & 408 & 389 & 348 & 411 & 354 & 402 & 460 & 457 & 3.932 & 45,59 \\
\hline 2 & 190 & 243 & 228 & 271 & 395 & 428 & 445 & 453 & 491 & 502 & 3.646 & 42,27 \\
\hline 3 & 41 & 34 & 44 & 52 & 101 & 111 & 114 & 100 & 159 & 137 & 893 & 10,35 \\
\hline 4 & 6 & 3 & 5 & 6 & 12 & 10 & 17 & 23 & 25 & 30 & 137 & 1,59 \\
\hline 5 & 0 & 0 & 1 & 0 & 1 & 2 & 1 & 2 & 2 & 6 & 15 & 0,17 \\
\hline 6 & 0 & 0 & 0 & 0 & 0 & 0 & 0 & 0 & 0 & 1 & 1 & 0,01 \\
\hline 10 & 0 & 0 & 0 & 0 & 0 & 0 & 0 & 0 & 0 & 1 & 1 & 0,01 \\
\hline Total & 576 & 644 & 686 & 718 & 857 & 962 & 931 & 980 & $\begin{array}{c}1.13 \\
7\end{array}$ & $\begin{array}{c}1.13 \\
4\end{array}$ & 8.625 & $\begin{array}{c}100,0 \\
0\end{array}$ \\
\hline
\end{tabular}

Fonte: Dados de pesquisa.

Análise da Tabela 3 permite verificar que há um considerável percentual de artigos, 45,59\%, procedentes de uma única instituição. Se desconsiderarmos os artigos de autoria única (que são 1.211), há 2.721 ou $31,54 \%$ de artigos intrainstitucionais, demonstrando, neste caso, que a proximidade física possui relevância nessas colaborações entre os autores; apesar de toda proximidade virtual que a Internet proporciona. Os artigos com instituição única e com duas instituições são responsáveis, então, por $87,86 \%$ dos artigos publicados, indicando que os pesquisadores da área de matemática preferem trabalhar em grupos mais fechados.

Artigos com três instituições diferentes aparecem em terceiro lugar, porém seu percentual corresponde a um quarto do percentual de artigos com duas instituições. A quantidade de artigos com quatro, cinco, seis e 10 instituições diferentes é relativamente baixo, ocupando 1,79\% do total.

Pela Tabela 3 fica mais evidente que a área de matemática não é muito inclinada a grandes colaborações entre instituições, tanto que ao longo de 10 anos, somente dois artigos tiveram mais de seis instituições colaboradoras. Também pode-se perceber que é maior a frequência daqueles artigos com uma e duas instituições. Os artigos com quatro instituições foram os que apresentaram maior taxa de crescimento anual, com média de 17,46\%. Já os artigos com apenas uma instituição foram os que apresentaram a menor taxa de crescimento anual, com média de 3,03\%. Os artigos com duas e três instituições apresentaram taxa de crescimento anual de $10,20 \%$ e $12,82 \%$, respectivamente. A seguir é possível visualizar melhor essas taxas de crescimento anuais:

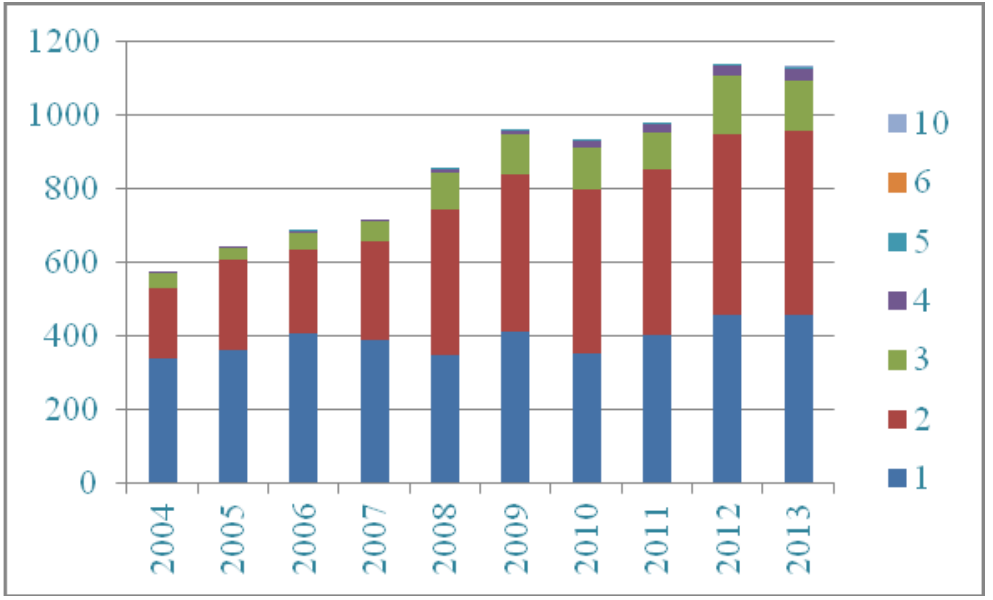

Gráfico 3: Número de instituições distribuídas por ano dos artigos brasileiros da área de matemática indexados na WoS entre 2004 e 2013

Fonte: Dados de pesquisa. 
Abaixo é feita a representação da colaboração entre essas instituições:

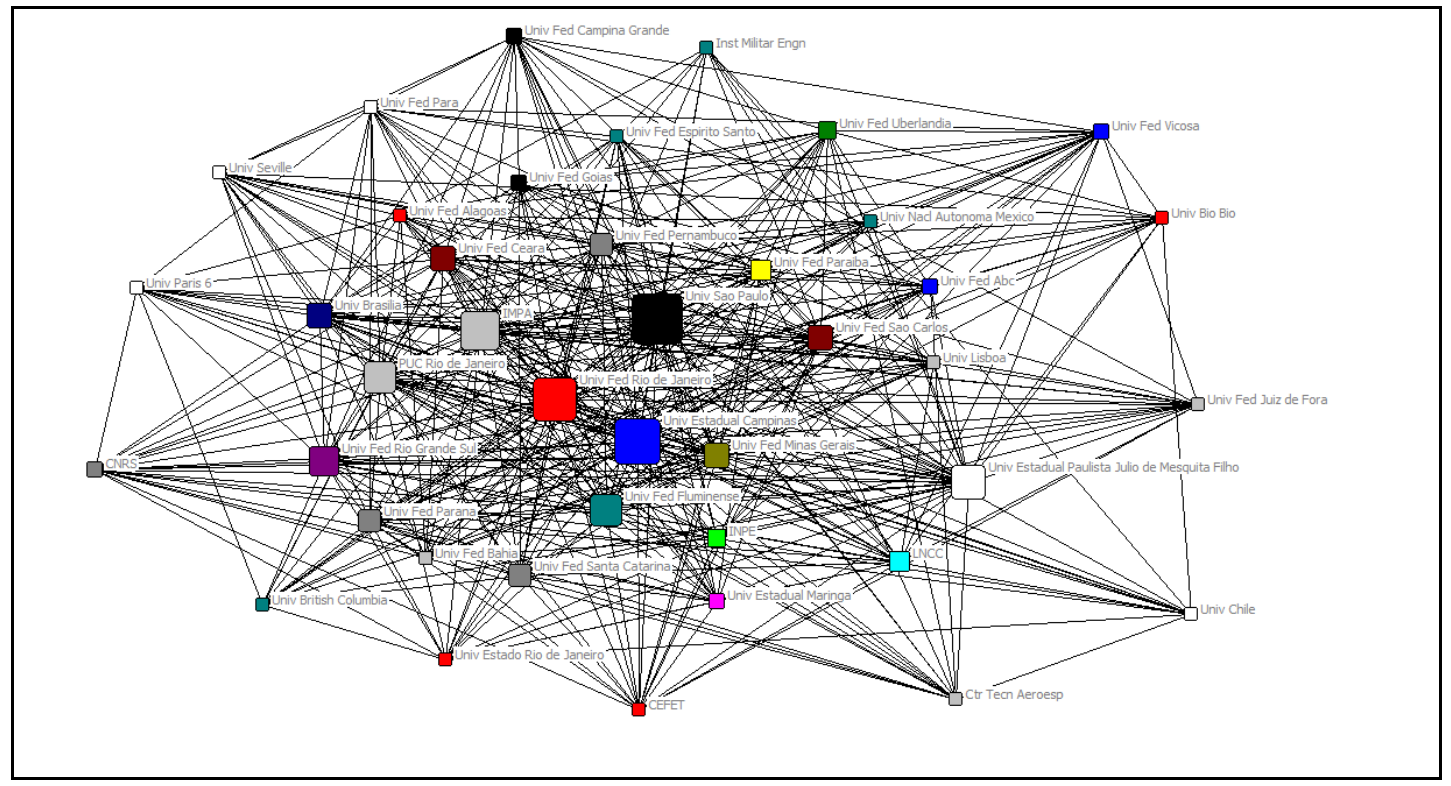

Figura 2: Mapa de colaboração entre instituições nacionais e internacionais mais produtivas entre os artigos brasileiros da área de matemática indexados na WoS entre 2004 e 2013 Fonte: Dados de pesquisa.

A construção da rede envolveu as 100 instituições com maior número de colaborações, totalizando 100 nós e 2.214 ligações, as quais variavam de no mínimo cinco ao máximo de 90 ligações. No entanto, para fins dessa análise, considerou-se aquelas instituições com no mínimo 20 ligações. Assim, chegou-se à rede apresentada acima, com 41 nós e 896 ligações. Os maiores clusters dessa rede são da Universidade de São Paulo - USP, Universidade Estadual de Campinas - UNICAMP e Universidade Federal do Rio de Janeiro - UFRJ.

Na rede analisada, o maior cluster é o da USP (nó na cor preta), com 90 ligações. A USP teve como maior colaboradora a UNICAMP (nó na cor azul), com 95 artigos em comum. Depois, a maior colaboradora foi a Universidade Estadual Paulista Júlio de Mesquita Filho - UNESP (nó na cor branca), com 85 artigos, seguida da Universidade Federal de São Carlos - UFSCAR (nó na cor marrom), com 56 artigos publicados juntos. Nota-se que os maiores colaboradores são todos do estado de São Paulo, denotando, nesse caso, que a proximidade física é um fator alto para a colaboração.

O segundo maior cluster é o da UNICAMP, com 81 ligações. Seus maiores colaboradores são a USP (conforme parágrafo anterior); a UNESP, com 54 artigos publicados juntos; e Universidade de Brasília - UNB (nó na cor azul-escuro) e UFRJ (nó na cor vermelha), com 29 artigos publicados cada uma. A relação de colaboração entre a UNICAMP com a UNESP pode ser em função da proximidade física entre as duas. Já a relação com a UNB parece estar ligada a participação docente no Programa de Pós-Graduação em Engenharia Mecânica da UNESP. E a relação de colaboração com a UFRJ parece estar ligada às relações de grupos de pesquisa, como, por exemplo, o Nano e Microfluidica e Microssistemas e o Engenharia Metabólica e de Bioprocessos.

Já o terceiro maior cluster é representado pela UFRJ, com 78 ligações. A UFRJ colabora mais com a Universidade Federal Fluminense - UFF (78 artigos), o Laboratório Nacional de Computação Científica - LNCC (43 artigos) e o IMPA (42 artigos). Todas as maiores instituições colaboradoras da UFRJ são do estado do Rio de Janeiro, indicando, da mesma forma que a USP, que a proximidade física pode ser uma facilitadora das colaborações neste meio científico.

Logo mais é apresentada a Tabela 4 com a distribuição dos autores por ano: 
Tabela 4: Número de autores distribuídos por ano dos artigos brasileiros da área de matemática indexados na WoS entre 2004 e 2013

\begin{tabular}{cccccccccccc}
\hline No autor & $\mathbf{2 0 0 4}$ & $\mathbf{2 0 0 5}$ & $\mathbf{2 0 0 6}$ & $\mathbf{2 0 0 7}$ & $\mathbf{2 0 0 8}$ & $\mathbf{2 0 0 9}$ & $\mathbf{2 0 1 0}$ & $\mathbf{2 0 1 1}$ & $\mathbf{2 0 1 2}$ & $\mathbf{2 0 1 3}$ & Total \\
\hline 1 & 106 & 117 & 109 & 111 & 127 & 128 & 109 & 122 & 139 & 143 & $\mathbf{1 . 2 1 1}$ \\
2 & 232 & 275 & 294 & 307 & 337 & 395 & 363 & 385 & 386 & 411 & $\mathbf{3 . 3 8 5}$ \\
3 & 163 & 180 & 179 & 196 & 253 & 312 & 287 & 298 & 362 & 350 & $\mathbf{2 . 5 8 0}$ \\
4 & 54 & 45 & 75 & 74 & 99 & 81 & 124 & 125 & 165 & 154 & $\mathbf{9 9 6}$ \\
5 & 12 & 18 & 19 & 23 & 25 & 30 & 29 & 40 & 47 & 47 & $\mathbf{2 9 0}$ \\
6 & 5 & 6 & 5 & 4 & 10 & 11 & 13 & 5 & 18 & 18 & $\mathbf{9 5}$ \\
7 & 1 & 0 & 4 & 1 & 2 & 2 & 2 & 4 & 11 & 8 & $\mathbf{3 5}$ \\
8 & 2 & 2 & 0 & 0 & 0 & 0 & 3 & 1 & 7 & 1 & $\mathbf{1 6}$ \\
9 & 1 & 1 & 1 & 2 & 2 & 3 & 1 & 0 & 0 & 0 & $\mathbf{1 1}$ \\
10 & 0 & 0 & 0 & 0 & 0 & 0 & 0 & 0 & 1 & 0 & $\mathbf{1}$ \\
11 & 0 & 0 & 0 & 0 & 1 & 0 & 0 & 0 & 1 & 0 & $\mathbf{2}$ \\
12 & 0 & 0 & 0 & 0 & 0 & 0 & 0 & 0 & 0 & 1 & $\mathbf{1}$ \\
13 & 0 & 0 & 0 & 0 & 0 & 0 & 0 & 0 & 0 & 1 & $\mathbf{1}$ \\
\hline 14 & 0 & 0 & 0 & 0 & 1 & 0 & 0 & 0 & 0 & 0 & $\mathbf{1}$ \\
\hline Total & $\mathbf{5 7 6}$ & $\mathbf{6 4 4}$ & $\mathbf{6 8 6}$ & $\mathbf{7 1 8}$ & $\mathbf{8 5 7}$ & $\mathbf{9 6 2}$ & $\mathbf{9 3 1}$ & $\mathbf{9 8 0}$ & $\mathbf{1 . 1 3 7}$ & $\mathbf{1 . 1 3 4}$ & $\mathbf{8 . 6 2 5}$ \\
\hline
\end{tabular}

Fonte: Dados de pesquisa.

A Tabela 4 mostra a predominância dos artigos escritos por grupos de dois e três autores, revelando a inclinação da área de matemática para trabalhos em grupos pequenos. Glänzel (2003) destaca que em matemática a colaboração não está associada a uma maior produtividade, ou seja, um grupo maior de autores trabalhando em colaboração não irão necessariamente publicar mais. Para Glänzel (2003), inclusive, na matemática a produtividade diminui com o crescimento da atividade de coautoria, como visto na tabela acima. Já Behrens e Luksch (2010) acreditam que o aumento da produtividade pode ser consequência do aumento de autoria múltipla, conforme resultados encontrados em sua pesquisa.

Os artigos com sete autores foram os que apresentaram maior taxa de crescimento anual, com $23,11 \%$, seguidos dos artigos com cinco autores, com taxa de $14,62 \%$. Os artigos com seis e quatro autores apresentaram respectivamente $13,66 \%$ e 11,04\% de taxa de crescimento anual. Já os artigos com dois e três autores, apesar de serem mais numerosos, apresentaram taxa de crescimento anual menor, de $5,88 \%$ e 7,94\% respectivamente. Os artigos com um autor somente apresentaram taxa de crescimento de $0,29 \%$, ou seja, um valor significativamente baixo; nesse ponto, indo em direção ao estudo de Behrens e Luksch (2010), os quais apontaram que o número de publicações de autoria única tem decrescido fortemente nas últimas décadas em matemática.

Percebe-se, de um modo geral, o crescimento, então, da colaboração sob forma da coautoria. A razão para esse desenvolvimento pode ser explicado por Glänzel (2003), o qual coloca que a colaboração na matemática tem sido influenciada pelo aumento da mobilidade dos cientistas e pela mudança nos seus padrões de comunicação.

Abaixo é feita a representação da colaboração entre esses autores: 


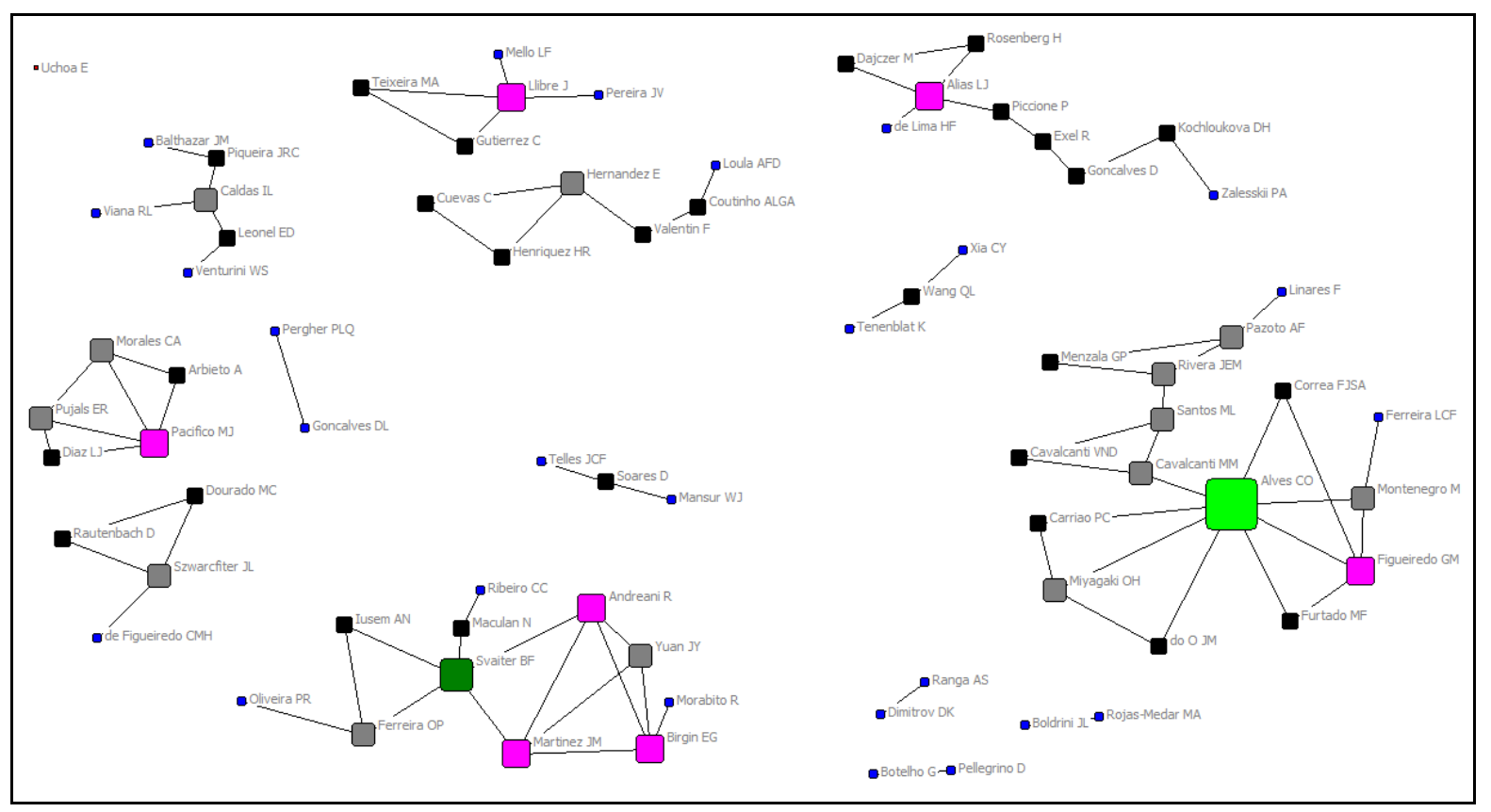

Figura 3: Mapa de colaboração dos principais autores dos artigos brasileiros da área de matemática indexados na WoS entre 2004 e 2013

Fonte: Dados de pesquisa.

A Figura 3 traz representado 77 nós dos autores mais produtivos, os quais apresentaram desde zero até oito ligações, totalizando 162 ligações. Os maiores clusters de autores brasileiros são de Alves CO e Svaiter BF.

O cluster de Alves CO (nó na cor verde limão) é o maior da rede, com oito ligações. Alves CO, da Universidade Federal de Campina Grande - UFCG, é o autor mais produtivo entre os 8.625 artigos analisados, com a publicação de 64 artigos de periódicos. Seus artigos são publicados em periódicos de Matemática e de Matemática, Aplicada. Nesse mesmo cluster também está Figueiredo GM (nó na cor rosa), da Universidade Federal do Pará - UFPA, com quatro ligações (segundo maior nó do cluster).

Fazem parte também desse cluster (nós na cor cinza): Montenegro M, da UNICAMP; Rivera JEM, do LNCC; Miyagaki OH, atualmente na Universidade Federal de Juiz de Fora - UFJF (também pertenceu à Universidade Federal de Viçosa - UFV); Pazoto AF, da UFRJ; Santos ML, da UFPA; e Cavalcanti MM, da Universidade Estadual de Maringá - UEM; cada um com três ligações. Montenegro M, Rivera JEM e Miyagaki $\mathrm{OH}$ estão entre os catorze autores mais produtivos nesta pesquisa no período analisado.

Nesse cluster ainda aparecem (nós na cor preta): Correa FJSA, da UFCG; Menzala GP, da UFRJ; Cavalcanti VND, da UEM; Carriao PC, da Universidade Federal de Minas Gerais - UFMG; do O JM, da Universidade Federal da Paraíba - UFPB; e Furtado MF, da UNB. Cada um destes nós possui duas ligações. Também fazem parte desse cluster (nós na cor azul): LInares F, do IMPA e Ferreira LCF, da UNICAMP. Ambos autores possuem uma ligação cada um.

A relação de colaboração entre Alves CO e Figueiredo GM provavelmente foi em decorrência de que Alves CO foi orientador de doutorado de Figueiredo GM. Já a parceria em trabalhos entre Alves $\mathrm{CO}$ e Montenegro M parece ter nascido quando Alves CO fez seu pós-doutorado na UNICAMP, local de trabalho de Montenegro M. Alves $\mathrm{CO}$ e Miyagaki $\mathrm{OH}$ podem ter desenvolvido trabalhos juntos porque participam no mesmo grupo de pesquisa, Equações Diferenciais não Lineares, da UFJF. Para Alves CO e Cavalcanti MM o desenvolvimento de uma parceria parece ter se desenvolvido a partir dos projetos de cooperação com os pesquisadores da Faculdade de Matemática e do Programa de Pós-graduação em Matemática do 
Centro em Desenvolvimento, da UFPA. Alves CO e Correa FJSA são colegas de universidade e participam do mesmo grupo de pesquisa, Equações Diferenciais Parciais, da UFCG. Já a colaboração entre Alves CO e Carriao PC parece decorrer do fato de pertencerem ao mesmo grupo de pesquisa, Equações Diferenciais Não Lineares, da UFJF. Alves CO e do O JM participam do mesmo Projeto/PVE № 407099/2013-1. Já Alves CO e Furtado MF foram contemporâneos de estudos na UNB, podendo ter iniciado nesse período uma relação de colaboração. Característica bastante importante nesse cluster de Alves CO é que todas suas relações diretas são com autores vinculados a instituições nacionais.

O cluster de Svaiter BF (nó na cor verde-escuro) é o segundo maior da rede, com cinco ligações. Svaiter BF, do IMPA, é o quinto autor mais produtivo, com 47 artigos publicados. Análise permitiu que se verificasse que seus artigos de periódicos são publicados em periódicos de Matemática; Matemática, Aplicada; Matemática, Aplicações Interdisciplinares; Pesquisa Operacional e Ciência de Gerenciamento; Ciências Sociais, Métodos Matemáticos; Automação e Controle de Sistemas; Ciência da Computação, Engenharia de Software; e Economia. Nesse mesmo cluster estão Martinez JM e Andreani R (nós na cor rosa), ambos da UNICAMP, com quatro ligações cada um.

Faz parte também desse cluster (nó na cor cinza) Ferreira OP, da Universidade Federal de Goiás - UFG, com três ligações. Nesse cluster ainda aparecem Maculan N, da UFRJ, e Iusem AN, do IMPA (nós na cor preta), com duas ligações cada.

A relação de colaboração entre Svaiter BF, Martinez JM, Andreani R, Ferreira OP e Iusem AN, pode ter sido em decorrência de terem feito parte do Núcleo de Excelência em Teoria e Métodos de Otimização Contínua (PRONEX - CNPq/FAPERJ 17/2009). Além disso, Ferreira OP fez seu mestrado no IMPA, local de trabalho de Svaiter BF e de seu outro colaborador, Iusem AN. Maculan N já atuou no Ministério da Educação e parece ter estreitas relações com o IMPA. Da mesma forma que o cluster de Alves CO, o cluster de Svaiter BF chama a atenção pelo fato de que seus maiores colaboradores serem todos ligados a instituições nacionais.

\section{CONSIDERAÇÕES FINAIS}

A pesquisa desenvolvida aqui permitiu, de um modo geral, que se desvelasse a área da matemática brasileira, através da análise da sua colaboração. Assim, análise dos indicadores de colaboração possibilitaram verificar que 85,95\% da produção de artigos se deu sob forma de coautoria, em sua maioria, internacional (principalmente entre o Brasil e mais um país). Constatou-se a preferência dos pesquisadores de matemática em trabalhar em grupos pequenos, formados por duas ou três pessoas. No entanto, o percentual de artigos de autoria única foi bem significativo, contribuindo para o histórico dos pesquisadores dessa área conhecidos pela preferência em trabalharem de forma isolada.

Os países que mais colaboraram com o Brasil foram os Estados Unidos e França. Já as instituições mais colaborativas foram a USP, UNICAMP e UFRJ, as quais também foram as que mais publicaram artigos. Em relação à coautoria entre autores, os maiores clusters formados são de pesquisadores da UFCG e da UNICAMP.

Assim, pode-se dizer que a área de matemática brasileira apresenta significativa internacionalização. Além disso, também pode-se dizer que a colaboração é predominante na sua produção científica. Talvez estes sejam os motivos pelos quais a área tem ganhado elevada importância não somente junto aos órgãos de fomento brasileiros, mas, principalmente, junto às instituições de pesquisa internacionais. 


\section{REFERÊNCIAS}

CASTANHA, Renata Cristina Gutierres; GRACIO, Maria Claudia Cabrini. Brazil's scientific production in mathematics: contribution to mainstream science (20022011). Journal Of Scientometric Research, New Delhi, v. 2, n. 3, p. 161-168, Set./Dec. 2013. Disponível em: <http://www.jscires.org/text.asp?2013/2/3/161/135405>. Acesso em: 01 ago. 2014.

CONSELHO NACIONAL DE DESENVOLVIMENTO CIENTÍFICO E TECNOLÓGICO CNPq. Portal CNPq. Disponível em: <http://www.cnpq.br/web/guest/view//journal_content/56_INSTANCE_0oED/10157/100343\#16061>. Acesso em: $11 \mathrm{dez}$. 2015.

COORDENAÇÃO DE APERFEIÇOAMENTO DE PESSOAL DE NÍVEL SUPERIOR CAPES. Documento de área 2013. Brasília, 2014. Disponível em:

<http://www.capes.gov.br/images/stories/download/avaliacaotrienal/Docs_de_ar ea/Matematica_Probabilidade_Estatistica_doc_area_e_comiss\%C3\%A3o_att08deout ubro.pdf>. Acesso em: 12 nov. 2014.

GLÄNZEL, Wolfgang. Bibliometrics as a research field: a course on theory and application of bibliometric indicators. [S.l.]: [s. n.], 2003. Disponível em:

<http://www.researchgate.net/profile/Wolfgang_Glaenzel/publication/242406991 _BIBLIOMETRICS_AS_A_RESEARCH_FIELD_A_course_on_theory_and_application_of_ bibliometric_indicators/links/0a85e536694a2980f9000000.pdf >. Acesso em: 30 ago. 2014.

LEYDESDORFF, Loet. The Challenge of Scientometrics: the development, measurement, and self-organization of scientific communications. 2. ed. Boca Raton: Universal, c2001.

MACIAS-CHAPULA, Cesar A. O papel da informetria e da cienciometria e sua perspectiva nacional e internacional. Ciência da Informação, Brasília, v. 27, n. 2, p.134-140, maio/ago. 1998. Disponível em:

<http://www.scielo.br/pdf/\%0D/ci/v27n2/macias.pdf>. Acesso em: 25 nov. 2011.

MOURA, Ana Maria Mielniczuk de et al. Panorama da produção conjunta entre Brasil e Espanha indexada na WoS entre 2006-2012: indicadores de atividade, especialização e colaboração. Informação \& Sociedade: Estudos, João Pessoa, v. 25, n. 1, p. 67-82, jan./abr. 2015.

SANZ-CASADO, Elias. 0 papel dos rankings para os novos desafios das universidades. Porto Alegre: UFRGS, 19 nov. 2014. Palestra ministrada aos professores e alunos da FABICO.

SIMIS, Aron et al. Mathematics: a testimony of its insertion in science, technology and innovation. In: CARVALHO, Antonio Carlos Campos de; CAMPOS, Diogenes de Almeida; BEVILACQUA, Luiz (Org.). Science in Brazil. Rio de Janeiro: Academia Brasileira de Ciências, 2002. p. 181-206.

VANZ, Samile Andréa de Souza. As redes de colaboração científica no Brasil: 2004-2006. 2009. 204 f. Tese (Doutorado) - Programa de Pós-Graduação em Comunicação e Informação, Faculdade de Biblioteconomia e Comunicação, UFRGS, Porto Alegre, 2009. Disponível em:

<http://www.lume.ufrgs.br/bitstream/handle/10183/17169/000711634.pdf?sequ ence $=1>$. Acesso em: 11 nov. 2014.

WEB OF SCIENCE - WoS. Site institucional. Disponível em: <http://thomsonreuters.com/en.html >. Acesso em: 31 maio 2015. 